\title{
EDITORIAL
}

\section{Editorial PIAM November 2021}

\author{
Eujin Pei ${ }^{1}$
}

Published online: 2 December 2021

(c) The Author(s), under exclusive licence to Springer Nature Switzerland AG 2021

Issue 04/2021 for the Progress in Additive Manufacturing Journal marks the first time that we have published 20 papers in a single issue. This is a milestone for us as it shows confidence in our growing number of submissions, which our high quality journal attracts authors from a wide range of disciplines within Additive Manufacturing and also from a diversity of geographic locations. We are also seeing papers submitted not just traditionally from universities, but also technological centres and companies. Through this, our readers will benefit from the breadth of topics and scientific depth of knowledge. We have also tried to publish (in some issues) with a more thematic approach where research on similar processes are grouped together and published in the same issue; and in other issues having a much broader scope of topics. For example, in this issue there are papers discussing Material Extrusion, Material Jetting, Binder Jetting and Multi-material Powder Bed Fusion processes. Other papers in this issue discuss the mechanical strength of specimens, post-processing methods such as "hot air polishing" by Heshmat; or using "sacrificial ice structures" by Moghanizadeh that are novel and pushing the boundaries of conventional norms of Additive Manufacturing. I hope you will enjoy reading these papers.

Personally, it has been a very long but meaningful year for me in academia, having taken on a new role as Associate Dean for the College of Engineering, Design and Physical Sciences at Brunel University London in the United Kingdom. Learning has no boundaries. And as I end this message, I take the opportunity to wish my colleagues from the Springer team, the Editorial Board and Advisory Board, our reviewers and readers a very Happy New Year 2022 with good health. Do write to me-I would love to receive feedback so that we can improve the journal for our readers.

Eujin Pei

eujin.pei@brunel.ac.uk

1 Brunel University, London, UK 